\title{
Planning and Deploying Transit Signal Priority in Small and Medium-Sized Cities: Burlington, Vermont, Case Study
}

\author{
Kleoniki Vlachou, University of Maryland, College Park \\ John Collura, University of Massachusetts, Amherst \\ Alex Mermelstein, Connecticut Department of Transportation
}

\begin{abstract}
Innovations in traffic signal systems have generated a great deal of interest in the provision of preferential traffic signal strategies and treatments for transit buses and other vehicles at signalized intersections in cities of all sizes. The primary objective of this paper is three fold: 1) to synthesize the literature of the lessons learned associated with planning and deploying transit signal priority (TSP) strategies in small and medium-sized cities; 2) to demonstrate the application of a micro-simulation model, VISSIM, to assess transit priority impacts in small and medium-sized communities where the required VISSIM input data are often limited; and 3) to present guidelines to aid traffic engineers and transit planners who are considering TSP strategies in small and medium-sized cities. An underlying aim of this paper is to recognize the differences in transit priority planning and deployment in small and medium-sized cities as compared to major metropolitan areas.
\end{abstract}




\section{Introduction}

Advances in traffic signal technologies and other factors have generated a great deal of interest in the provision of preferential traffic signal strategies and treatments for transit buses and other vehicles at signalized intersections in cities of all sizes. To plan and deploy such signal priority strategies and treatments safely and efficiently, careful analyses should be conducted using fundamental traffic engineering and transit management and operating principles. Based on these principles and other considerations, this paper focuses on providing guidance to aid traffic engineers and transit planners in planning and deploying signal priority strategies in small and medium-sized cities.

\section{Objective of the Paper}

The primary objective of this paper is three fold: 1) to synthesize the literature of the lessons learned associated with planning and deploying transit signal priority (TSP) strategies in small and medium-sized cities; 2) to demonstrate the application of a micro-simulation model, VISSIM, to assess transit priority impacts in small and medium-sized communities where the required VISSIM input data is often limited; and 3) to present guidelines to aid traffic engineers and transit planners who are considering TSP strategies in small and medium-sized cities. The application of VISSIM is part of a case study on the formulation and evaluation of alternative transit signal strategies in Burlington, Vermont (2000 urbanized area population: 105,365). The impacts of concern in the simulation include bus travel time and delay and side-street queue length.

An overarching aim of the paper is to assist state DOTs and highway and transit agencies in the design and implementation of signal priority strategies for transit buses in concert with other preferential signal treatments, such as those currently in place and being planned for emergency response, including fire and rescue services. Finally, an underlying aim is to recognize the differences in transit priority in small and medium-sized cities as compared to major metropolitan areas. These differences relate to both technical and institutional issues.

\section{TSP Study Results and Lessons Learned}

Numerous studies have been conducted in small and medium-sized cities in the United States and Europe to evaluate the impacts of transit priority deployments. These studies fall into two categories. The first includes studies that used simula- 
tion to evaluate the anticipated impacts , and the second includes studies where field tests were conducted. The studies that used simulation are summarized in Table 1 (Kamdar 2004; Deshpande et al. 2003; Chang et al. 2003; Dion et al. 2004; Collura et al. 2004; Garrow and Mechemehl 2007; Ova and Smadi 2001). The simulation models most frequently used include VISSIM, TRANSYT, NETSIM, INTEGRATION and SCOOT.

Bus travel time is the most commonly-used measure to assess the impact of transit priority. As shown in Table 1, bus travel time reduction varies significantly among the studies. In a study in Arlington, Virginia (Chang et al. 2003), bus travel time decreased by almost one percent, and in another study in Fairfax, Virginia (Deshpande et al. 2003), the decrease was nearly three percent. On the other end, a study in Fargo, North Dakota (Ova and Smadi 2001) estimated the bus travel time decrease to be 14 percent. Other measures used in some of these studies are side-street queue lengths and side-street person delay, overall vehicle-delay, and stopped delay/vehicle, which estimate the impact of transit priority strategies to non-transit traffic. In most cases, the impact was not significant, excluding the study in Fargo, where side-street person delay increased by 14 percent.

The field studies conducted in small and medium-sized cities are summarized in Table 2 (Ahn et al. 2006; Collura et al. 2004; Zhang 2001; Fox et al. 1998; Deshpande 2003). Measures such as vehicle/person delay, cross street delays, and side-street effects most often showed few significant impacts.

The findings in the studies outside the U.S. are consistent with those within the U.S. and provide additional evidence regarding the beneficial impacts of TSP without significantly impacting overall traffic. 


\section{Table 1. Results of Transit Priority Studies Using Simulation}

\begin{tabular}{|c|c|c|}
\hline Simulation Studies & Measure & Result \\
\hline \multirow{3}{*}{$\begin{array}{l}\text { Fairfax, VA-U.S.1 VISSIM } \\
\text { (Deshpande } \\
\text { et al. 2003) }\end{array}$} & Bus Travel Time & 2.64\% decrease \\
\hline & Time Reliability & $3.61 \%$ improvement \\
\hline & $\begin{array}{l}\text { Average Queue Length on } \\
\text { Side Street }\end{array}$ & $\begin{array}{l}1.28 \mathrm{ft} \text { increase (less than one } \\
\text { car length); not significant }\end{array}$ \\
\hline \multirow{3}{*}{$\begin{array}{l}\text { Arlington, VA Columbia Pike } \\
\text { Blvd INTEGRATION } \\
\text { (Chang et al. 2003) }\end{array}$} & Bus Travel Time & $0.9 \%$ decrease \\
\hline & Arrival Reliability & $3.2 \%$ improvement \\
\hline & Overall Vehicle-Delay & $1 \%$ increase \\
\hline \multirow{2}{*}{$\begin{array}{l}\text { Arlington, VA Columbia Pike } \\
\text { Blvd SCOOT/INTEGRATION } \\
\text { (Dion et al. 2004) }\end{array}$} & Bus Travel Time & $6 \%$ decrease \\
\hline & Overall Person-Delay & $8 \%$ increase \\
\hline \multirow{2}{*}{$\begin{array}{l}\text { Bremerton, WA (Collura et } \\
\text { al. 2004) }\end{array}$} & Bus Travel Time & $10 \%$ decrease \\
\hline & Stopped Delay/Vehicle & Not significant \\
\hline $\begin{array}{l}\text { Ann Arbor, Michigan } \\
\text { NETSIM/TRANSYT-7F } \\
\text { (Collura et al. 2004) }\end{array}$ & Bus Travel Time & $\begin{array}{c}6 \% \text { decrease } \\
\text { (for a single bus) }\end{array}$ \\
\hline $\begin{array}{l}\text { Austin, Texas NETSIM } \\
\text { (Garrow and Machemehl } \\
\text { 2007) }\end{array}$ & Bus Travel Time & $\begin{array}{l}11 \% \text { decrease (optimized } \\
\text { lower cycle length), } 10 \% \\
\text { decrease (phase splitting) }\end{array}$ \\
\hline \multirow{3}{*}{$\begin{array}{l}\text { Fairfax, VA-U.S.1 VISSIM } \\
\text { (Kamdar 2004) }\end{array}$} & Transit Travel Time & $0.8 \%$ to $4 \%$ decrease \\
\hline & Control Delay & $5 \%$ to $16 \%$ decrease \\
\hline & Side-Street Queue Length & $1.23 \%$ increase \\
\hline \multirow{3}{*}{$\begin{array}{l}\text { Fargo, ND } \\
\text { (Ova and Smadi 2004) }\end{array}$} & Bus Travel Time & $14 \%$ decrease \\
\hline & Bus Stopped Delay & $38 \%$ decrease \\
\hline & Side-Street Person Delay & $14 \%$ increase \\
\hline
\end{tabular}




\section{Table 2. Results of Transit Priority Field Studies}

\begin{tabular}{|c|c|c|}
\hline Field Studies & Measure & Result \\
\hline \multirow{3}{*}{$\begin{array}{l}\text { St. Cloud, } \\
\text { Stearns County, MN } \\
\text { (Collura et al. 2004) }\end{array}$} & Bus Delay & $43 \%$ decrease \\
\hline & Average Bus Occupancy & 24 \\
\hline & Bus Travel Time & 13 to $18 \%$ decrease \\
\hline \multirow{2}{*}{$\begin{array}{l}\text { Anne Arundel County, } \\
\text { MD } \\
\text { MDSHA Opticom } \\
\text { (Collura et al. 2004) }\end{array}$} & $\begin{array}{l}\text { Auto Travel Time-Same Direc- } \\
\text { tion }\end{array}$ & $9 \%$ decrease \\
\hline & $\begin{array}{l}\text { Auto Travel Time- } \\
\text { Opposing Direction }\end{array}$ & 4 to $5 \%$ increase \\
\hline \multirow[t]{2}{*}{$\begin{array}{l}\text { Tacoma, WA-Pierce } \\
\text { Transit Agency Opticom } \\
\text { (Collura et al. 2004) }\end{array}$} & Bus Travel Time & $\begin{array}{l}5.8-9.7 \% \text { decrease (green extension); } \\
8.2 \% \text { decrease (green extension and/ } \\
\text { or early green) }\end{array}$ \\
\hline & Side Street Impacts & Not significant \\
\hline \multirow{2}{*}{$\begin{array}{l}\text { Charlotte, NC/OPTICOM } \\
\text { (Express Buses) } \\
\text { (Collura et al. 2004) }\end{array}$} & Bus Travel Time & 4 minute decrease \\
\hline & Cross Street Delays & Not acceptable \\
\hline \multirow{2}{*}{$\begin{array}{l}\text { Toulouse, France } \\
\text { (Zhang 2001) }\end{array}$} & Bus Travel Time & 11 to $14 \%$ decrease \\
\hline & General Traffic Travel Time & Not significant change \\
\hline $\begin{array}{l}\text { Strasbourg, France } \\
\text { (Zhang 2001) }\end{array}$ & Transit Vehicle Travel Time & 4 to $5 \%$ decrease \\
\hline \multirow{2}{*}{$\begin{array}{l}\text { Vicenza, Italy Opticom } \\
\text { (Zhang 2001) }\end{array}$} & Bus Travel Time & $23.8 \%$ decrease \\
\hline & Bus Travel Speed & $30 \%$ increase \\
\hline \multirow[t]{2}{*}{$\begin{array}{l}\text { Swansea, England } \\
\text { SCOOT(Zhang 2001) }\end{array}$} & Bus Travel Time & $\begin{array}{l}2 \% \text { decrease (passive priority); } 11 \% \\
\text { decrease (greenextension/red trun- } \\
\text { cation); no change (green extension) }\end{array}$ \\
\hline & Non Transit Vehicle Delay & $\begin{array}{l}17 \% \text { increase (passive priority); } \\
7 \% \text { increase (green extension/red } \\
\text { truncation); } 15 \% \text { increase (green } \\
\text { extension) }\end{array}$ \\
\hline \multirow{2}{*}{$\begin{array}{l}\text { Leeds, England } \\
\text { SPOT (Fox et al. 1998) }\end{array}$} & Bus Travel Time & $10 \%$ decrease \\
\hline & Non Transit Vehicle Travel Time & No Change \\
\hline \multirow{2}{*}{$\begin{array}{l}\text { Stuttgart, Germany } \\
\text { (Deshpande 2003) }\end{array}$} & Light Rail Transit Delay & $50 \%$ decrease (conditional priority) \\
\hline & Private Vehicle Delay & Minimal \\
\hline $\begin{array}{l}\text { Zurich, Switzerland } \\
\text { (Deshpande 2003) }\end{array}$ & Bus Waiting Time & $\begin{array}{l}\text { Zero (at } 90 \% \text { of signalized } \\
\text { intersections) }\end{array}$ \\
\hline \multirow{2}{*}{$\begin{array}{l}\text { Fairfax, VA, U.S.1 (Ahn et } \\
\text { al. 2006) }\end{array}$} & Transit Vehicle Travel Time & $3 \%$ to $6 \%$ decrease \\
\hline & Intersection Delay & $9 \%$ to $23 \%$ decrease \\
\hline
\end{tabular}




\section{Simulation Analysis}

\section{Transit Priority Scenarios and Evaluation Measures}

For the purpose of this research, two different areas of Burlington were examined with the use of the micro-simulation model, VISSIM. The first is Route 15, a fourlane arterial that connects the city of Burlington with the suburbs. The other is the Old North Route, a loop located in downtown Burlington. For the first area, data were available and coded in Sychro and were imported easily in VISSIM. This was not the case for the second location, which is more typical for small and medium-sized cities. In small and medium-sized cities, such Synchro files may not be readily available and thus may require field data collection, which was done in this research. In both cases, the number of routes chosen to deploy TSP is small, constituting another difference between metropolitan areas and small and medium-sized cities. In small and medium-sized cities, planners should be selective in choosing a small number of routes along which TSP may be appropriate, as opposed to large metropolitan areas where there could be many more routes along which TSP might be considered.

Route 15. Two TSP scenarios along Route 15 were evaluated for this research. One included a 10-second green extension for the AM buses in the inbound direction, assumed to be operating under existing conditions, including approximately 30-minute headways. In the second scenario, the inbound buses also may request a 10-second green extension, but the headways were changed to 15 minutes, reflecting the interest among local stakeholders to improve the frequency of bus service along selected bus routes in the region. For this research, only green extensions were considered because the ridership is relatively small compared to the automobile and red truncation is very disruptive and would not be adequately justified. Four major categories of evaluation measures were employed in this simulation analysis: 1) travel time for the bus and vehicle; 2 ) delay to the bus and vehicle; 3 ) waiting time for outbound buses; and 4) side-street queue length. The definitions of these measures are summarized in Table 3 , as defined in the VISSIM Manual (2005).

The average values for each evaluation measure were calculated based on 20 runs for the first scenario and 8 runs for the second scenario. A statistical analysis using the Student's t-test was first conducted for the absolute values of the samples, followed by a second statistical analysis on the difference of the values. More details on this analysis are presented in Vlachou (2007). 


\section{Table 3. Summary of Definitions of the Measures of Effectiveness Used}

\begin{tabular}{|l|l|}
\hline Measure & Description \\
\hline $\begin{array}{l}\text { Travel Time } \\
\text { (sec) }\end{array}$ & $\begin{array}{l}\text { The time required for a vehicle to travel between the first } \\
\text { cross-section (start) of the network and the second cross- } \\
\text { section (destination), including waiting or dwell times. }\end{array}$ \\
\hline Delay (sec) & $\begin{array}{l}\text { The average total delay per vehicle is computed for every vehicle } \\
\text { completing the travel time section by subtracting the theoreti- } \\
\text { cal (ideal) travel time from the real travel time. The theoretical } \\
\text { travel time is the time that would be reached if there were no } \\
\text { other vehicles and no signal controls or other stops in the net- } \\
\text { work (reduced speed areas are taken into account). The delay } \\
\text { does not include passenger stop times at transit stops. However, } \\
\text { the loss time caused by acceleration or deceleration because of } \\
\text { such a stop remains part of the delay time. }\end{array}$ \\
\hline $\begin{array}{l}\text { Bus Waiting } \\
\text { Time (sec) }\end{array}$ & $\begin{array}{l}\text { All events when a transit vehicle is stopped, excluding passen- } \\
\text { ger interchange stops and stops at stop signs. }\end{array}$ \\
\hline $\begin{array}{l}\text { Side Street } \\
\text { Queue Length } \\
\text { (feet) }\end{array}$ & $\begin{array}{l}\text { The maximum queue counted from the location of the queue } \\
\text { counter on a link upstream to the final vehicle that is in queue } \\
\text { condition. }\end{array}$ \\
\hline
\end{tabular}

The average bus travel times for Scenario 1 and Scenario 2 comparing to the base case (i.e., without priority vs. with priority) are shown in Figure 1(a) and 1(b), respectively. In Scenario 1, it appears that the reduction in average bus travel time with priority is almost five percent, and in Scenario 2, this reduction is almost six percent. It should be noted that in the first scenario, the $t$-statistic of the absolute values shows that the difference of the means in not statistically significant but that the $t$-statistic of the differences of the values shows that the difference is significant. For the second scenario, both t-tests showed that the difference of the means is not statistically significant.

The computed vehicle travel time is for those vehicles that move in the same direction as the buses that have the ability to request priority. The comparison of the average travel time of vehicles in each scenario is shown in Figure 1(a) and 1(b). In Scenario 1 , the reduction in vehicle travel time is estimated to be less than one-half 
percent and for Scenario 2 about six percent, neither of which, based on the t-test analysis, proves to be statistically significant.

The values of bus delay for Scenario 1 and Scenario 2 with and without transit priority are shown in Figure 1(a) and 1(b), respectively. The results suggest that in Scenario 1 there is a 14.2 percent reduction of bus delay for buses with priority and a reduction of 16.5 percent in Scenario 2 when priority is provided. The $t$-test analysis shows that the difference of the average values for the first scenario is statistically significant and that the second scenario difference of the average values was not statistically significant. The t-test for the difference of the rates of change showed that the difference of the means is statistically significant.

The average vehicle delay computed for each scenario also is presented in Figure $1(a)$ and $1(b)$. The reduction in delay of the vehicles that travel in the same direction as the buses that get priority is about one percent in scenario 1 and about nine and one-half percent in the Scenario 2 . In both scenarios, the statistical analysis showed that the differences of the means are not statistically significant.

The outbound buses travel in the non-peak direction and do not get priority. The average bus waiting time outbound is shown in the Figure 1(a) and 1(b). In both scenarios, there appears to be an increase in the waiting time of the outbound line when priority is provided. This increase was about 12.4 percent for Scenario 1 and four percent for Scenario 2. For both scenarios, it was shown that these increases are not statistically significant.

As indicated here, the inbound line is in the peak direction and gets priority. The average waiting times of these buses are depicted in Figures 1(a) and 1(b). In Scenario 1 , the reduction in the rate of change in the waiting time estimated is to be about 27.9 percent ; this reduction in Scenario 2 is about 27.3 percent. In both scenarios, the estimates are statistically significant.

Figure 2(a) presents the maximum queue lengths computed for Scenario 1. For Scenario 1, the change of queue length appears to be relatively small, ranging from a four and one-half percent increase to a seven percent decrease. The $t$-test shows that the differences are not statistically significant. The maximum queue lengths for Scenario 2 are presented in Figure 2(b). For Scenario, 2 the change fluctuates from a 19.7 percent increase to an approximately 2 percent decrease. The $t$-test here also shows that the difference is not significant. 


\section{Scenario 1}

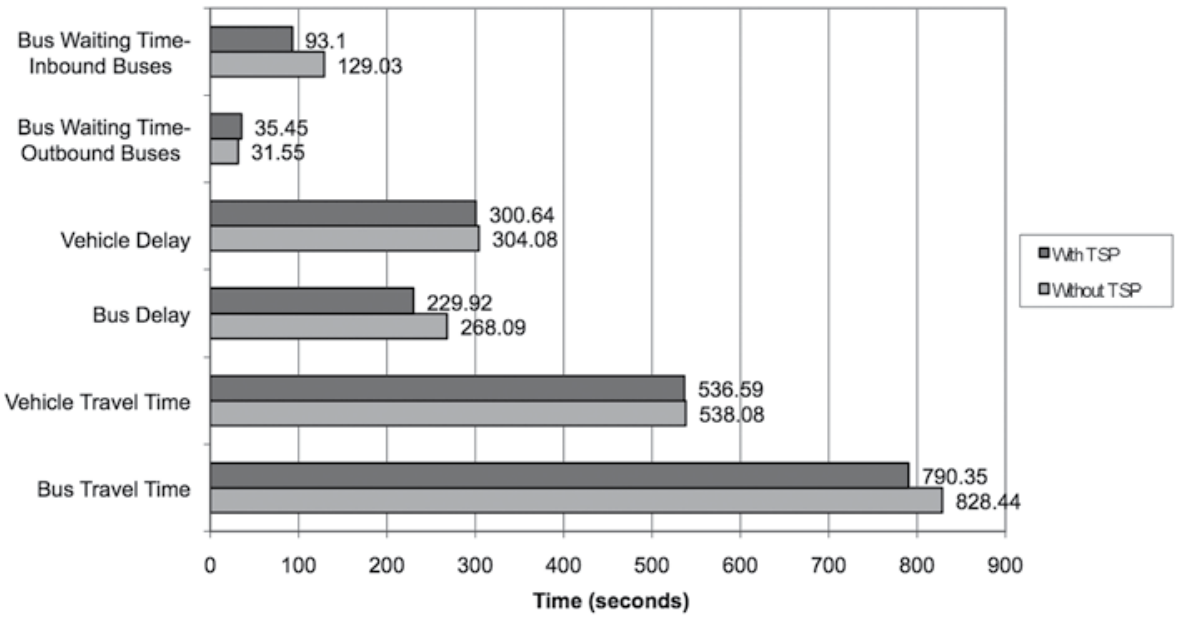

(a)

\section{Scenario 2}

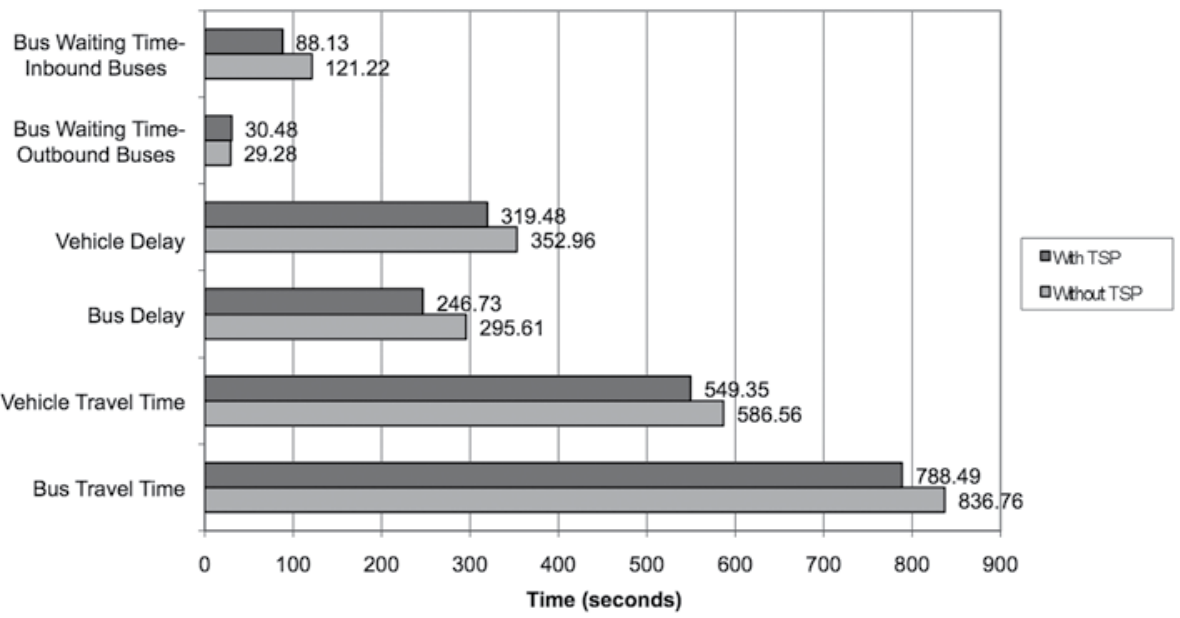

(b)

Figure 1. Evaluation Measures for Scenario 1 and Scenario 2 (in seconds) 


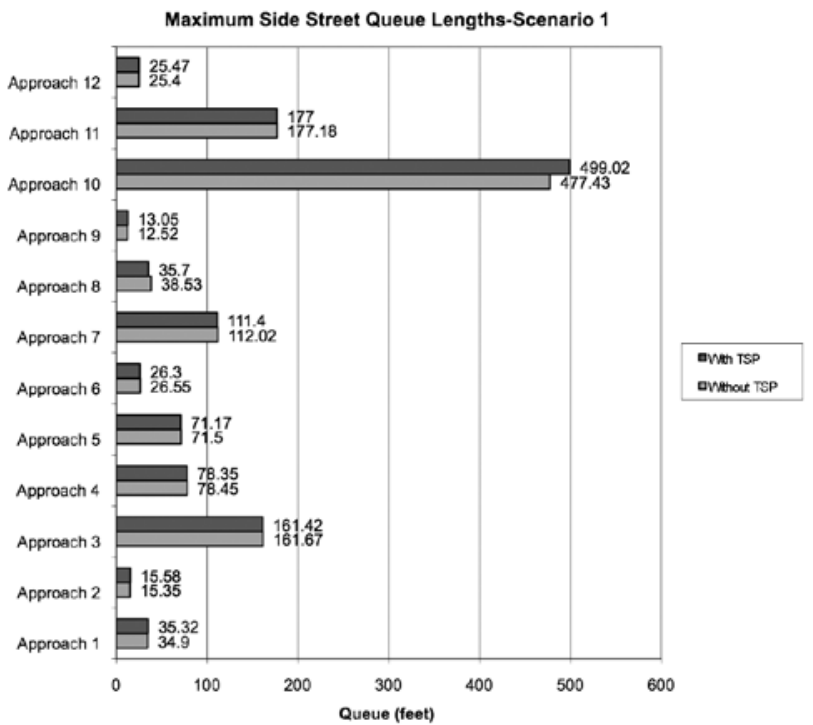

(a)

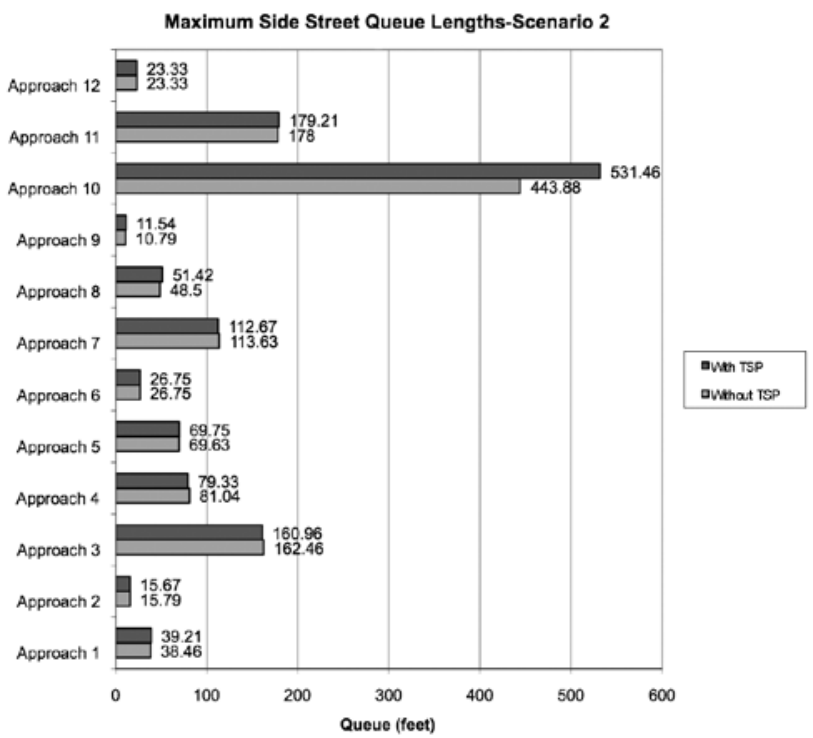

(b)

Figure 2. Maximum Side Street Queue Length (in feet) for Scenario 1 and Scenario 2 
Old North Route. For the purpose of this research, two transit priority scenarios along the Old North Route were evaluated. One included a 10-second green extension for the AM buses traveling around the entire loop under existing schedules. Also for this corridor, only green extensions were considered because adding red truncation would create great disruption, which is not justified by the relatively low ridership. In the second scenario, it was assumed that all bus stops of the nearside type would be relocated to the farside, reflecting the notion that farside stop locations may reduce travel time. Two evaluation measures were employed in this simulation analysis: 1) travel time for the bus and 2) delay to non-transit vehicles.

The average values for each evaluation measure were calculated based on 20 runs for each scenario. A statistical analysis using the Student's t-test was used to examine statistical significance. The results of the simulation analyses are summarized below. Further details are contained in Mermelstein (2007).

The average values of bus travel times to traverse the entire bus route are presented in Figure 3(a) for the base case (no priority) and the two scenarios. As can be observed, Scenario 1 shows a seven percent reduction in travel time, as compared to the base case and Scenario 2, which show an approximately two and one-half percent reduction, as compared to Scenario 1 . The $t$-test analysis revealed that average travel times for the base and Scenario 1 are significantly different from each other, while the t-test did not show a statistically significant difference between travel times for Scenarios 1 and 2 .

Figure 3(b) compares the average values of total delay for each scenario and the base case. There is a less than one percent decrease of total delay for other vehicles for Scenario 1 as compared to base scenario. There is a less than one percent decrease of total delay for other vehicles when comparing scenario 1 and scenario 2. Based on the t-test, the differences in delays to non-transit vehicles in the base case versus scenario 1 and scenario 2 versus scenario 1 were not statistically significant. 
Average Bus Travel Time

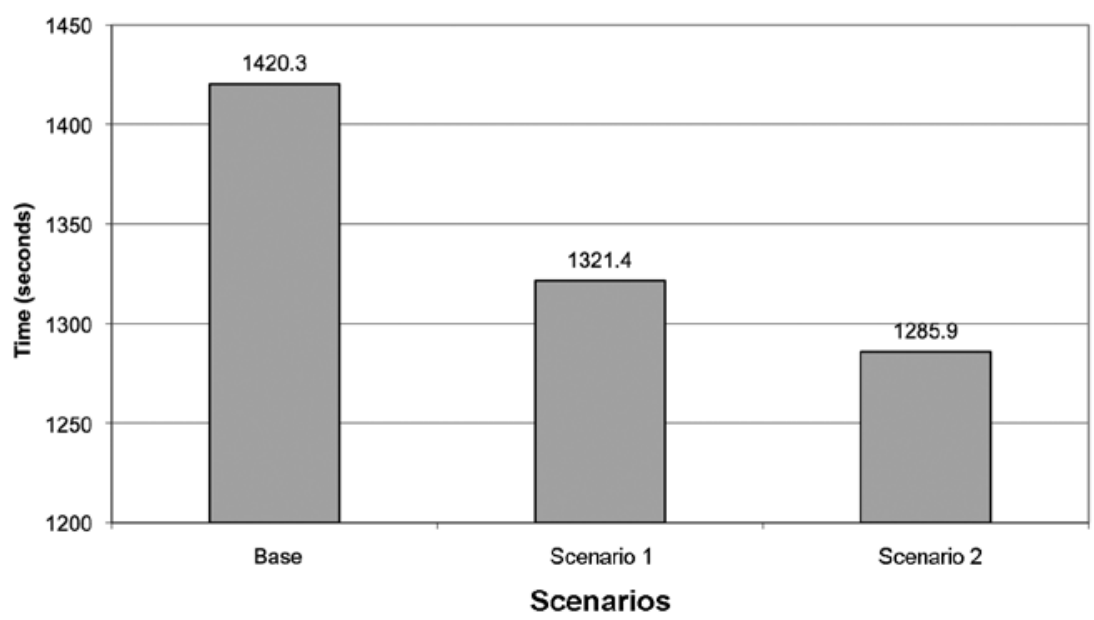

(a)

Average Total Vehicle Delay

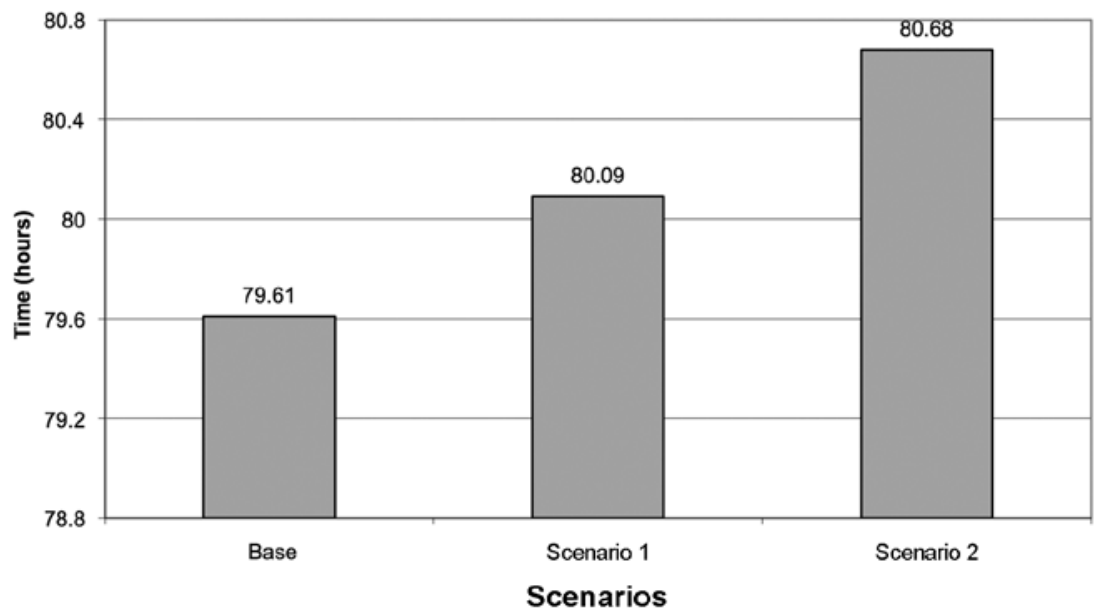

(b)

Figure 3. Average Bus Travel Times (in seconds) and Average Total Vehicle Delay (in hours) Along Old North Route 


\section{Simulation Results}

From the simulation analyses, some preliminary conclusions can be drawn. The major results suggest that transit priority may aid in improving overall bus travel time along Route 15 and the Old North Route and that these results are generally consistent with the results reported in other TSP simulation analyses as well as before-and-after field studies. Also, there is no significant evidence that the 10-second green extension along Route 15 creates added waiting time delay to the buses that move along the opposite direction and do not get priority. Finally, there is no significant evidence that the 10-second green extensions along Route 15 and the Old North Route increases delay for the non-transit traffic along the side streets off Route 15 and the overall traffic on the Old North Route.

\section{Guidelines}

One of the objectives of this paper is the development of a set of guidelines to assist traffic engineers and transit planners in the planning and deployment of TSP strategies. In the analysis of transit priority concepts, transit priority is described as a form of traffic signal control strategy provided to facilitate the flow and passage of transit buses. Transit priority requests often are conditional and may, for example, be granted based on one or more conditions such as the absence of a pedestrian phase, the presence of a green interval, and a prescribed level of bus occupancy or degree of bus lateness. The guidelines are divided into two sections: 1) Planning, and 2) Deployment. These guidelines should be of interest to state and local traffic engineers and public transit planners and operators who are contemplating the implementation of a transit priority strategy.

\section{Planning}

\section{Institutional Issues, Local Needs Assessment, and System Objectives and}

Requirements. Planning for a transit priority system is not a trivial task. A variety of institutional issues and local concerns must be addressed, ranging from the integration of transit priority into existing and potentially incompatible emergency vehicle preemption systems, to the identification of the important stakeholders, to the assessment of priority system needs and the formulation of local transit priority objectives and requirements (Collura et al. 2004; IBI 2006). These objectives and requirements provide the basis for an evaluation of transit priority strategies using either simulation models or field tests. 
Pre-Deployment Impact Analysis. As part of planning, traffic engineers, transit planners, and other stakeholders should take steps to ensure that a local impact analysis is conducted to assess the anticipated consequences of alternative transit priority strategies under consideration. Among those consequences may be the impact on transit schedule adherence as well as impacts on traffic flow and vehicular and pedestrian safety. This local impact analysis may include field tests and/or the use of microscopic simulation analysis as presented before.

Based on a review of literature, the impacts of transit priority have been shown to have both positive and negative impacts in more than a dozen actual transit priority deployment projects in the U.S. and abroad. Moreover, simulation analyses reported in the literature review have produced results generally consistent with the impacts actually experienced in the project deployments.

Traffic Flow. There is significant evidence reported in TSP issues that the implementation of transit priority strategies may reduce travel times for transit vehicles. However, another expected impact may be delay to all other vehicles. Most transit priority projects have been deployed in the U.S. only within the past eight or nine years, and results from operational field test evaluations and simulation analyses are difficult to compare across the board because performance measures are not well defined in a standardized framework. Moreover, different transit priority strategies including green extensions only and green extension in combination with red truncation and other tactics, yield different impacts.

It should also be stressed that traffic simulation models may be a cost-effective means to analyze the impact of transit priority on traffic flow. As part of this research project, the VISSIM simulation model was used to assess impacts of a green-extension-only strategy on both transit and non-transit vehicles. Results indicated that bus service reliability could be improved, travel time would possibly diminish, and non-transit vehicle delay would likely be minimal. It also should be pointed out that the transit priority strategy might have a varying level of impact on transit and other vehicles. A green-time extension has also been determined by others to provide benefits to buses with no travel time impact to other users (Collura et al. 2004). However, a green extension in combination with red truncation (i.e., recall) may negatively impact non-transit vehicles, depending on the frequency of bus service. It is further recommended that a strategy consider the specific conditions that influence the corridor or area of interest. These conditions may include frequency and direction of travel for vehicles requesting priority, roadway characteristics, travel demand, presence and frequency of pedestrian 
phases, transition strategy, cycle characteristics, and intersection spacing and progression strategy (Obenberger and Collura 1998). The use of different types of priority, such as queue jumping and phase re-servicing, in addition to green extension, may be necessary to match the status of the intersection in order not to affect signal coordination (Hood et al. 1995).

Safety for Pedestrians. Pedestrian fatalities typically account for more than 10 percent of motor vehicle deaths nationwide annually. In terms of accident locations, approximately one-third of accidents involving pedestrians have occurred at intersections (Zegeer and Seiderman 1994). It is suggested that a safety audit be conducted during the planning of transit priority systems, especially at locations near college campuses and in downtown areas. This audit should review the potential impacts that transit priority strategies might have on pedestrian safety. This audit should review the historical accident data within the area of interest, the length of pedestrian cycles based on the age and other demographics of the local population, the location of residential housing and retail activities, the location and placement of bus stops and pull-off areas, and the distance between bus stop locations.

Economic Analysis. It is strongly recommended that an economic analysis be performed prior to transit priority deployment to identify and estimate the fixed and recurring costs associated with priority investments. Recurring costs should include, for example, costs of an equipment maintenance agreement, as described below. ITS projects such as transit priority typically may have a short service life, lower upfront investment costs, and higher operating costs than traditional physical infrastructure projects. Since the cash flow profiles of ITS and traditional investments are radically different and the time value of money for ITS investments may not be that important, it has been argued that traditional benefit-cost analysis may not be appropriate and that a multi-criteria analysis approach should be used (Leviakangas and Lahesmaa 2002). It is suggested that life-cycle cost analysis be employed and an attempt be made to look at all life-cycle capital and operational costs within a larger economic analysis framework.

Financing. A financial plan for transit priority system deployment needs to be developed. This plan will identify funding sources to support capital investments and defray operating and maintenance costs. Funding is available from federal, state, and local sources, such as Congestion Management and Air Quality (CMAQ) and other programs in the SAFETEA-LU legislation of 2005. It should also 
be stressed that such public funding sources may include transportation agencies as well as local fire and rescue departments.

\section{Deployment}

Procurement. While it has been suggested that transit priority systems can be procured using standard procurement processes, there are special considerations that need to be taken into account. Lessons learned from past ITS procurements and procurement experiences were used to provide insights into the identification of system objectives and requirements and preparation of requests for proposals and proposal evaluation.

Identification of Systems Objectives and Requirements. The procurement process begins with the identification of project objectives and requirements. As mentioned above, a clear understanding of the project scope of work objective is required of all stakeholders and participants to manage expectations and to preclude misunderstanding later in the process. Technological limitations also must be understood. A common frame of reference and a common definition of terms will need to be developed and adhered to. The proposed system objectives and requirements will then be translated into technical and operational requirements for vendors to develop into a fully-functional system. Sound technical specifications are a prerequisite for success. Vaguely-defined requirements will result in confusion and will necessitate negotiation with the contractor to settle differences.

RFP Preparation/Proposal Evaluation. A Request for Proposals (RFP) defines the project scope of work and system objectives and requirements, provides the technical and operational performance requirements, outlines the compliance requirements, and defines the performance period. It is suggested that a single integrator be responsible for design, procurement of components, system integration, installation, testing of the project, and user training.

Pre-Installation Site Survey. A pre-installation survey by the contractor(s) is highly recommended. As part of this on-site survey, the contractor should determine the impact of roadway geometry, bus stop placements, line of sight restrictions, pedestrian crossing volumes, and existing equipment to the system design. In addition, detector placement must be carefully sited to avoid putting a bus in a dilemma zone when the traffic signal turns amber. Detector placement and installation will need to consider the impacts of bus speed, length of green extension, and intersection width as well as the location of bus stops. For example, for 
a bus traveling at $15 \mathrm{mph}$ ( $22 \mathrm{fps}$ ) with a maximum green extension of 10 seconds through an intersection width of $40 \mathrm{ft}$, a detection distance of approximately 180 $\mathrm{ft}$ provides sufficient time to allow the bus to clear the dilemma zone.

System Installation. The typical priority system has three major subsystem components, including in-vehicle subsystems, roadside subsystems, and center subsystems. Each subsystem has its own installation challenges. In-vehicle subsystems consist of those component parts of the system that are installed on the vehicle. For example, a simple priority system may consist of the emitter and its power system and microprocessor system. More complex systems may include a vehicle location device such as a Global Positioning System (GPS) locator and Automatic Passenger Counters (APCs). Roadside subsystems are those parts of the system that reside outside the designated vehicles. Typically, they would include detectors mounted in the vicinity of the traffic signals and power sources that service the detectors, microprocessors, and communications equipment collocated with the traffic signal controller boxes. Center subsystems are those items of equipment that must interface with the central traffic signal management system and the transit management system.

It is recommended that the contractor be responsible for quality control throughout the installation process. The contractor should be required to provide installation drawings for approval. In addition, the contractor should be required to present a prototype installation of every subsystem and complete operational testing of all prototype installations. The contractor also should provide for review of site-specific installation specifications tailored to the physical characteristics of each site.

Evaluation. System evaluations during deployment provide a means to assess whether a priority system meets its intended objectives. The evaluation process should consist of the following elements: 1) an evaluation frame of reference, 2) evaluation planning, 3) evaluation implementation, and 4) potential evaluation spin-offs (Casey and Collura 1994).

The evaluation frame of reference provides a context for the evaluation. It defines the project objectives, external influences, local issues, and site characteristics. The evaluation plan outlines what should be measured (the impacts) and how impacts might be measured (measurement techniques). Evaluation implementation outlines evaluation plan execution, data collection, and analysis. For additional guidance on the design of ITS project evaluations, see the U.S. DOT's Joint Program Office website (2009). 
A major product of the evaluation is an assessment of system objectives and impacts, including benefits, costs, and other consequences. Transit priority system objectives may relate to transit service reliability and efficiency and other traffic impacts. In addition, the priority system evaluation should consider assessing broader impacts related to interoperability, maintainability, reliability, expandability, affordability, institutional and organizational issues, and human factors.

An institutional issue where differences exist between planning and evaluating TSP strategies in small and medium-sized areas compared to large metropolitan areas relates to differences in staffing. Typically, there is limited staffing in small and medium-sized cities as compared to large metro areas. Thus, in small and medium-sized cities, it is important to attempt to keep the planning and evaluation of TSP alternatives simple and easy to carry out and to employ user-friendly simulation software (VISSIM) with relatively minimal data input requirements and data requirements.

Finally, it should be stressed that continuous evaluations should be conducted as soon as possible during deployment. Evaluations provide a means to measure the performance of the system against the measures used, and the results supply agencies in other metropolitan areas with useful information regarding deployment results, challenges, and lessons learned.

\section{Summary, Conclusions and Recommendations}

Innovations in traffic signal technology and other factors have increased the interest in TSP in small and medium-sized cities. The primary goal of this paper is to assist regional agencies and local jurisdictions in considering the use of traffic signal systems and technologies to implement TSP strategies for buses. The research includes an evaluation of the impacts, merits, and limitations associated with alternative TSP strategies and a review of the lessons learned in communities similar to those in Vermont where such strategies have been deployed. An underlying aim of the project is to assist transit planners and public agencies in planning and deploying signal priority strategies for transit buses in concert with other preferential signal treatments such as traffic signal preemption strategies. The coordination of TSP and preemption strategies for multiple types of vehicles is of utmost importance to preserve safety, facilitate emergency response, enhance traffic flow, and improve overall mobility. 
The results of transit priority system deployments in the U.S. and abroad suggest that transit priority in small and medium-sized urban areas may reduce transit travel time and may lead to improvements in transit schedule adherence and other aspects of transit performance without major negative impacts on overall traffic flow. Also, the results of the preliminary simulation analyses suggest that transit priority may aid in improving overall bus travel time along Route 15 and the Old North Route and that these results are generally consistent with the results reported in other TSP simulation analyses as well as before-and-after field studies, as reported in the literature review. In addition, the simulation analyses suggest that there is no significant evidence that a 10 -second green extension increases delay for the non-transit traffic along the streets intersecting Route 15 and the overall traffic on the Old North Route.

Finally, the guidelines developed should be employed by local jurisdictions, transportation agencies, and public safety agencies in the planning and design of transit priority strategies and treatments along signalized arterials.

An underlying aim of this paper is to recognize the differences in transit priority planning and deploying in small and medium-sized cities as compared to major metropolitan areas. The differences between planning and evaluating TSP strategies in small and medium-sized areas compared to large metropolitan areas relate to both technical and institutional issues. Technical issues have to do with data availability and transit usage. For example, typically in large metropolitan areas, input data required by simulation models such as VISSIM are readily available and, in fact, may be coded in Synchro files, which are easily accommodated by VISSIM. In small and medium-sized cities, such Synchro files may not be readily available and thus require field data collection, which was done in this research and described to guide transit planners in small and medium-sized cites.

Also, in small and medium-sized cities where transit ridership is relatively small as compared to automobile and other forms of travel, transit planners should be very selective, as in this research, in choosing the TSP strategy, e.g., green extension only. Planners in small and medium-sized areas also should be selective in choosing a small number of routes along which TSP may be appropriate, as opposed to in large metropolitan areas where there could be many more routes along which TSP (including a red truncation) might be considered.

An institutional issue relates to differences in staffing. Typically, there is limited staffing in small and medium-sized cities, compared to large metropolitan areas. Thus, in small and medium-sized cities, it is important to attempt to keep the 
planning and evaluation of TSP alternatives simple and easy to carry out and to employ user-friendly simulation software (VISSIM) with relatively minimal data input requirements and data requirements.

Recommendations for future research:

- Carry out additional simulation analyses considering other priority strategies, including longer green extensions and multiple $A M, P M$, and mid-day peak analysis periods. As part of future simulation analyses, sensitivity analyses should be included considering different bus headways, bus stop types and locations, and fare collection methods.

- Conduct a small-scale transit priority field test in conjunction with the additional simulation analyses. As part of the field test, a set of transit priority objectives and evaluation criteria should be used to assess the performance of the priority system. These objectives and criteria should relate to bus service reliability, bus efficiency, and other impacts on non-transit traffic and overall traffic flow. As part of a transit priority field test, it is recommended that a contractor (e.g., the system/equipment vendor or a third party) be responsible for quality control throughout the system installation process. The contractor should be required to provide roadside equipment installation drawings for approval. In addition, the contractor should be required to present a prototype installation of each subsystem including roadside and in-vehicle components and complete operational testing of all prototype components as necessary. Finally, a maintenance agreement with a contractor should be established to deal with system/equipment challenges and malfunctions (if any) during the field test period.

\section{References}

Ahn, K., H. Rakha, and J. Collura. 2006. Evaluation of green extension transit signal priority strategies using portable GPS receivers. Presented at the 85th Annual Meeting of the Transportation Research Board, Washington, D.C.

Casey, R. F., and J. Collura. 1994. Advanced Public Transportation Systems: Evaluation Guidelines. Final Report, Office of Technical Assistance, Federal Transit Administration, Washington, D.C. 
Chang, J., J. Collura, F. Dion, and H. Rakha. 2003. Evaluation of service reliability impacts of traffic signal priority strategies for bus transit. Transportation Research Record 1841: 23-31.

Collura, J., H. Rakha, and J. Gifford. 2004. Guidelines for the Planning and Deployment of Emergency Vehicle Preemption and Transit Priority Strategies. Prepared for Virginia DOT, Fairfax, Virginia.

Deshpande, V. 2003. Evaluating the impacts of transit signal priority strategies on traffic flow characteristics: Case study along U.S.1, Fairfax County, Virginia. Master's thesis, Virginia Polytechnic Institute and State University.

Deshpande, V., J. Collura, D. Teodorovic, and S. Tignor. 2003. Evaluating the impacts of transit signal priority strategies on traffic flow characteristics. Paper prepared for submission to the Transportation Research Board.

Dion, F., H. Rakha, and Y. Zhang. 2004. Evaluation of transit signal priority benefits along a fixed-time signalized arterial. Journal of Transportation Engineering 103(3): 294-303.

Fox, K., C. Haibo, F. Montgomery, M. Smith, and S. Jones. 1998. Selected Vehicle Priority in the UMTC Environment. Institute of Transportation Studies, University of Leeds. http://www.its.leeds.ac.uk/projects/spruce/utmc1rev.html, accessed 23 June 2009.

Garrow, M., and R. Machemehl. 2007. Development and Evaluation of Transit Signal Priority Strategies. Southwest Region University Transportation Center, University of Texas at Austin, Report No. SWUTC/97/472840-00068-1.

Hood, W., T. Hicks, and L. Singer. 1995. Light rail preemption of traffic signals: A question of balance. Paper presented at the Seventh National Conference on Light Rail Transit, November 12-15, in Baltimore, Maryland.

IBI Group. 2006. Regional Traffic Signal Pre-emption /Priority Standard Evaluation, Chittenden County Metropolitan Planning Organization.

Kamdar, V. 2004. Evaluating the transit signal priority impacts along the U.S. 1 corridor in Northern Virginia. Master's thesis, Virginia Polytechnic Institute and State University.

Leviakangas, P. and J. Lahesmaa. 2002. Profitability evaluation of intelligent transportation system investments. Journal of Transportation Engineering 128(3): 276-286. 
Mermelstein, A. 2007. Transit signal priority in small urban areas: An evaluation of the impacts along the Old North End Loops bus route in Burlington, VT. Master's thesis, University of Massachusetts-Amherst.

Obenberger, J. and J. Collura. 1998. Transition strategies to exit preemption control: State-of-the-practice assessments. Transportation Research Record 1748: 72-79.

Ova, K., and A. Smadi. 2001. Evaluation of Transit Signal Priority Strategies for SmallMedium Cities. www.ndsu.edu/ndsu/ugpti/DPpdf/DPQ142.pdf, accessed 16 June 2009.

PTV Planning Transport Verkehr AG. 2005. VISSIM 4.10 User Manual.

Smith, H., B. Hemily, and M. Ivanovic. 2005. Transit Signal Priority: A Planning and Implementation Handbook. Intelligent Transportation Society of America. http://www.itsa.org/itsa/files/pdf/TSPHandbook2005.pdf, accessed 16 June 2009.

U.S. Department of Transportation 2009. Research and Innovative Technology Administration. http://www.its.dot.gov/index.htm, accessed 24 June 2009.

Vlachou, K. 2007. Evaluation of transit signal priority along Route 15 in Burlington, VT. Master's thesis, University of Massachusetts-Amherst.

Zegeer, C. V. and C. B. Seiderman. 1994. Designing for Pedestrians, The Traffic Safety Toolbox. Institute of Transportation Engineers.

Zhang, Y. 2001. An evaluation of transit signal priority and SCOOT adaptive signal control. Master's thesis, Virginia Polytechnic Institute and State University.

\section{About the Authors}

KLEONIKI VLACHOU (kvlachou@umd.edu) is a Ph.D. candidate and graduate researcher at the University of Maryland, College Park. She received her Diploma in Civil Engineering from the National Technical University of Athens and her master's degree in Transportation from the University of Massachusetts, Amherst. Her research interests include air transportation, public transportation, and Intelligent Transportation Systems.

Dr. John Collura (collura@ecs.umass.edu) is Professor of Civil Engineering at the University of Massachusetts in Amherst and serves as the Director of the 
UMass Transportation Center. From 1998 to 2004, he was a Professor and Director of the Advanced Transportation Systems and Infrastructure Programs at Virginia Tech. He has been teaching and conducting transportation research for more than 25 years and is a registered professional engineer. He has taught courses in public transportation planning and operations, intelligent transportation systems, and transportation systems analysis and has published more than 100 papers in refereed journals and conference proceedings. He earned his Ph.D. in civil engineering at North Carolina State University.

AleX Melmestein (amermelstein@state.ct.us) is employed with the Connecticut Department of Transportation Traffic Operations Section. He earned his M.S. in Civil Engineering and and B.S. in Economics at the University of Massachusetts at Amherst. 